\title{
Pulsed Focused Nonlinear Acoustic Fields from Clinically Relevant Therapeutic Sources in Layered Media: Experimental Data and Numerical Prediction Results
}

\author{
Tamara KUJAWSKA \\ Department of Ultrasound, Institute of Fundamental Technological Research, Polish Academy of Sciences \\ Pawińskiego 5B, 02-106 Warsaw, Poland; e-mail: tkujaw@ippt.pan.pl
}

(received March 1, 2012; accepted May 18, 2012)

\begin{abstract}
In many therapeutic applications of a pulsed focused ultrasound with various intensities the finiteamplitude acoustic waves propagate in water before penetrating into tissues and their local heating. Water is used as the matching, cooling and harmonics generating medium. In order to design ultrasonic probes for various therapeutic applications based on the local tissue heating induced in selected organs as well as to plan ultrasonic regimes of treatment a knowledge of pressure variations in pulsed focused nonlinear acoustic beams produced in layered media is necessary. The main objective of this work was to verify experimentally the applicability of the recently developed numerical model based on the TimeAveraged Wave Envelope (TAWE) approach (WóJCIK et al., 2006) as an effective research tool for predicting the pulsed focused nonlinear fields produced in two-layer media comprising of water and tested materials (with attenuation arbitrarily dependent on frequency) by clinically relevant axially-symmetric therapeutic sources. First, the model was verified in water as a reference medium with known linear and nonlinear acoustic properties. The measurements in water were carried out at a $25^{\circ} \mathrm{C}$ temperature using a $2.25 \mathrm{MHz}$ circular focused (f/3.0) transducer with an effective diameter of $29 \mathrm{~mm}$. The measurement results obtained for 8-cycle tone bursts with three different initial pressure amplitudes varied between $37 \mathrm{kPa}$ and $113 \mathrm{kPa}$ were compared with the numerical predictions obtained for the source boundary condition parameters determined experimentally. The comparison of the experimental results with those simulated numerically has shown that the model based on the TAWE approach predicts well both the spatial-peak and spatial-spectral pressure variations in the pulsed focused nonlinear beams produced by the transducer used in water for all excitation levels complying with the condition corresponding to weak or moderate source-pressure levels. Quantitative analysis of the simulated nonlinear beams from circular transducers with $k a \gg 1$ allowed to show that the axial distance at which sudden accretion of the 2nd or higher harmonics amplitude appears is specific for this transducer regardless of the excitation level providing weak to moderate nonlinear fields. For the transducer used, the axial distance at which the 2nd harmonics amplitude suddenly begins to grow was found to be equal to $60 \mathrm{~mm}$. Then, the model was verified experimentally for two-layer parallel media comprising of a $60-\mathrm{mm}$ water layer and a $60-\mathrm{mm}$ layer of 1.3-butanediol (99\%, Sigma-Aldrich Chemie GmbH, Steinheim, Germany). This medium was selected because of its tissue-mimicking acoustic properties and known nonlinearity parameter $B / A$. The measurements of both, the peak- and harmonic-pressure variations in the pulsed nonlinear acoustic beams produced in two-layer media (water/1.3-butanediol) were performed for the same source boundary conditions as in water. The measurement results were compared with those simulated numerically. The good agreement between the measured data and numerical calculations has shown that the model based on the TAWE approach is well suited to predict both the peak and harmonic pressure variations in the pulsed focused nonlinear sound beams produced in layered media by clinically relevant therapeutic sources. Finally, the pulsed focused nonlinear fields from the transducer used in two-layer media: water/castor oil, water/silicone oil (Dow Corning Ltd., Coventry, UK), water/human brain and water/pig liver were predicted for various values of the nonlinearity parameter of tested media.
\end{abstract}

Keywords: therapeutic ultrasound, circular focused transducers, pulsed nonlinear acoustic pressure beams, layered media, numerical modeling and experiments.

\section{Introduction}

A majority of currently used therapeutic ultrasonic techniques employs circular focused transducers gener- ating pulsed acoustic pressure waves with various intensity penetrating into tissues after passing through a water layer. The water layer prior tissues is used as the matching and cooling medium. Besides, water is 
a weakly attenuating medium, therefore the secondary waves with harmonic frequencies are there easily generated causing an increase of nonlinear waveform distortion. An absorption of the nonlinearly distorted tone bursts increases with increasing of number of harmonics generated. A concentration of energy of pulsed nonlinear waves inside tissues and increase of their absorption lead to a local heating of tissues in the area of the beam focus. In order to design ultrasonic probes for various therapeutic applications based on a tissue heating as well as to select the safe ultrasonic regime of treatment a prediction of both the pressure variations in the pulsed focused nonlinear acoustic beams and heat sources distributions induced in tissues due to these fields is required (KUJAWSKA et al., 2011b).

However, there is a lack of papers published (according to the knowledge of authors), which address the pulsed nonlinear focused beams from axiallysymmetric acoustic sources in layered media with arbitrary dependence of attenuation on frequency. This work presents experimental verification of the numerical model based on the Time-Averaged Wave Envelope (TAWE) approach by comparing the measured spatial peak- and harmonic- pressure variations in the pulsed nonlinear sound beams from a circular focused transducer in two-layer media: water/tested material with those simulated numerically. A description of the TAWE approach to the numerical solution of the second order nonlinear differential wave equation for axially-symmetric sources is presented in (WóJCIK et al., 2006). The numerical solver, being the computer implementation of the model, accounts for the effects of diffraction, absorption, nonlinear interaction of harmonics as well as of transmission and reflection at the media interfaces.

This paper is organized as follows. First, both the source and media boundary condition parameters, required for the nonlinear propagation model as the input data, are described. Then, detailed description of the experimental setup and measurement methodology in water and in two-layer media is given. Next, the results of measurements in water (as a reference medium with known linear and nonlinear acoustic properties) as well as in two-layer media: water/1.3-butanediol are presented and compared with those simulated numerically for the source boundary conditions determined experimentally. Finally, pressure distributions of the harmonic components in the pulsed focused nonlinear sound beams produced in two-layer media comprising of water and tested fluids (castor oil, DC-710 silicone oil, Dow Corning Ltd., Coventry, UK) or tissues (pig liver, human brain) were predicted for various nonlinearity parameters of the tested medium.

\section{Numerical model}

The numerical model used in this work was presented in (WóJCIK et al. 2006; 2008). The model de- scribes (in frequency domain) nonlinear waveform distortion of a pulsed finite-amplitude sinusoidal wave generated from an axially-symmetric acoustic source and propagating in a dissipative fluid with an attenuation arbitrarily dependent on a frequency. The model developed is computationally efficient due to application of the Time-Averaged Wave Envelope (TAWE) approach to the numerical solution of the second order differential nonlinear wave equation for sources with axial symmetry. The model was extended to multilayer media via introduction to the model equations the terms accounting for the effects of transmission and reflection at the media interfaces. The computer implementation of the extended model was a new effective research tool for investigation and quantitative analysis of the pulsed nonlinear fields generated from the single-element circular planar or focused acoustic sources in layered attenuating media with arbitrary frequency-dependence of attenuation.

The model requires a number of input parameters related to the source geometry and working conditions as well as to the acoustic properties of the media of propagation. The source boundary condition parameters required for the model include the transducer centre frequency $f_{0}$, effective radius $a_{\text {eff }}$, focal length $F$, source-pressure amplitude $P_{0}$, radiating aperture apodization function $P_{0}(r)=P_{0} f(r)$, initial tone burst waveform $P(t)$ and pulse repetition frequency (PRF).

The source boundary condition parameters used in numerical simulations were determined experimentally by using the comparison-fitting method. The radial pressure distribution $P(r)$ was measured in water by the needle hydrophone at the axial distance of $5 \mathrm{~mm}$ from the transducer face and fitted to the simulated one by adjusting the source-pressure amplitude $P_{0}$, effective radius $a_{\text {eff }}$ and index $m$ in the radiating aperture apodization function introduced to the model in a form of the analytical function

$$
P_{0}(r)=P_{0}\left|1-\left(r / a_{\mathrm{eff}}\right)^{m}\right| .
$$

For the transducer used, the optimal apodization function, which produced in water at the axial distance of $5 \mathrm{~mm}$ the radial pressure distribution best fitted to the measured one, was found when the effective radius of the source was equal to $14.5 \mathrm{~mm}$ and exponent $m$ was equal to 10 for all excitation levels used.

The initial tone burst pressure-time waveform $P(t)$ used in the numerical simulations was also determined by the comparison-fitting method and introduced to the numerical model in the form of polynomial function:

$$
P(t)=\left[1-\left|\left(t-t_{c}\right) /\left(t_{b}-t_{e}\right)\right|^{q}\right] \cdot \sin \left[\omega\left(t-t_{c}\right)\right]
$$

for $t_{b} \leq t \leq t_{e} ; P(t)=0$ for $t \notin\left(t_{b}, t_{e}\right)$. Here $t_{c}, t_{b}$ and $t_{e}$ are dimensionless times determining the midpoint, 
Table 1. The acoustic parameters of tested media.

\begin{tabular}{|l|c|c|c|c|c|c|c|}
\hline \multicolumn{1}{|c|}{ Medium } & $\begin{array}{c}\text { Density } \\
{\left[\mathrm{kg} / \mathrm{m}^{3}\right]}\end{array}$ & $\begin{array}{c}\text { Sound velocity } \\
{[\mathrm{m} / \mathrm{s}]}\end{array}$ & $\begin{array}{c}\text { Absorption coefficient } \\
{\left[\mathrm{Np} /\left(\mathrm{m} \cdot \mathrm{Hz}^{b}\right)\right]}\end{array}$ & $b$ & $B / A$ & $\begin{array}{c}T \\
{\left[{ }^{\circ} \mathrm{C}\right]}\end{array}$ & Literature \\
\hline Distilled water & 997 & 1497 & $0.025 \cdot\left(10^{-6}\right)^{b}$ & 2 & 5.2 & 25 & {$[2]$} \\
\hline 1.3-butanediol & 1005 & 1530 & $1.27 \cdot\left(10^{-6}\right)^{b}$ & 2 & 10.5 & 25 & {$[3]$} \\
\hline Castor oil & 969 & 1452 & $5.8 \cdot\left(10^{-6}\right)^{b}$ & 1.667 & $5-13$ & 25 & {$[9]$} \\
\hline DC-710 Silicone oil & 1102 & 1378 & $7.3 \cdot\left(10^{-6}\right)^{b}$ & 1.79 & $5-13$ & 25 & {$[9]$} \\
\hline Pig liver & 1060 & 1590 & $7.8 \cdot\left(10^{-6}\right)^{b}$ & 1 & $5-9$ & 25 & {$[4]$} \\
\hline Human brain & 1040 & 1562 & $5 \cdot\left(10^{-6}\right)^{b}$ & 1.08 & 7 & 37 & {$[4]$} \\
\hline
\end{tabular}

beginning and end of the initial tone burst, respectively, $t$ is dimensionless time and $q$ is integer related to the envelope function of the tone burst. The space and time were normalized regarding to $2 \pi$ (see Eq. (2) in WóJCIK et al., 2006). The pressure-time waveform $P(t)$ that best reproduced the waveform of the initial tone burst measured on the acoustic axis close to the transducer surface comprised of 8 cycles and enabled determining the optimal initial tone burst waveform for the index $q$ equal to 8 .

The acoustic parameters of media required for the model include their density $\rho$, sound velocity $c$, attenuation coefficient $\alpha_{1}$, power index $b$ determining the dependence of attenuation on frequency $\alpha(f)=\alpha_{1} \cdot f^{b}$ and nonlinearity parameter $B / A$. The density, sound velocity and frequency-dependent attenuation law used in numerical simulations for tested media considered in this work were available from the literature and are quoted in Table 1 . The value of the nonlinearity parameter $B / A$ for these media was assumed to be varying between 5 and 13 . These values cover the range of the $B / A$ typical for most physiological fluids and soft tissues.

Similarly to the commonly used KZK nonlinear propagation model the model based on the TAWE approach has limitations. The limitation of the model used was determined in (BAKER et al., 1988; NACHEF et al., 1995) showing that the model equations provide very good prediction of the measured sound fields from planar circular sources in water when the sourcepressure level is weak to moderate, i.e. when the ratio of the shock formation (discontinuity) distance $\left(l_{D}=\rho_{0} c_{0}^{2} \lambda_{0} /(2+B / A) \pi P_{0}\right)$ to the Rayleigh distance $\left(R_{0}=\pi a^{2} / \lambda_{0}\right)$ is larger than $0.3\left(l_{D} / R_{0}>0.3\right)$. In case of a planar transducer with a diameter and centre frequency the same as for the transducer used here fulfillment of this condition means that the pressure amplitude on its surface should be above the value providing the linear propagation mode but below $214 \mathrm{kPa}$. Physically, this condition means that a shock is formed only in the transition- or far-field region of the acoustic beam. However, therapeutic applications of ultrasound involve the focused beams in which shock is formed in the near-field region of the beam. So, the experimental investigation of focused beams in water and in two-layer media (comprising of water and the tested medium) provides an opportunity to verify the applicability of the numerical model based on the TAWE approach as the effective research tool for predicting of the peak- and harmonic- pressure variations in the pulsed focused nonlinear beams, produced by therapeutic transducers in layered attenuating media.

\section{Experimental setup for measurements in water}

First, the model was verified in water as a reference medium with known linear and nonlinear acoustic properties. All measurements were carried out at a $25^{\circ} \mathrm{C}$ temperature using a $2.25 \mathrm{MHz}$ frequency, circular focused (f-number 3) transducer with a $29 \mathrm{~mm}$ effective diameter. The experimental setup used for the measurements is shown in Fig. 1. The Arbitrary Function Generator (Ritec Advanced Measurement System RAM-10000, Warwick, RI, USA) produced 2.25 MHz, 8-cycle tone bursts with $1 \mathrm{kHz}$ pulse repetition frequency (PRF). This signal was applied to the piezoelectric transducer used (Olympus-NDT Inc.,

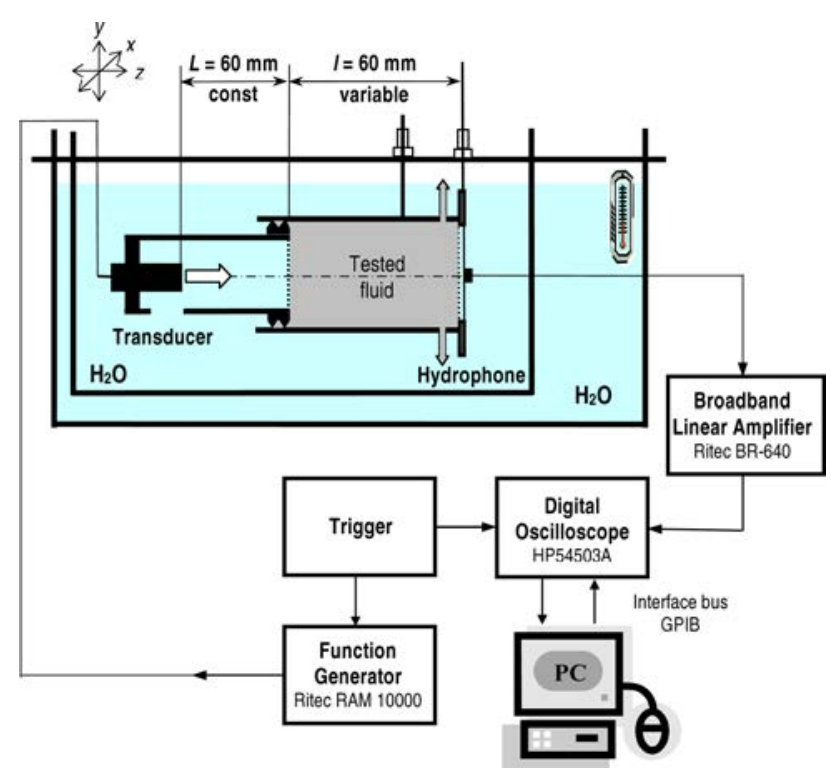

Fig. 1. Schematic diagram of the experimental setup. 
Waltham, MA, USA) which generated tone bursts with the initial pressure amplitude varied from $37 \mathrm{kPa}$ to $117 \mathrm{kPa}$. The pressure-time waveforms were recorded by the $0.513 \mathrm{~mm}$, broadband bilaminar, PVDF membrane hydrophone (Unisyn, formerly Sonora, SN S5259, Longmont, CO) with an integrated preamplifier. The hydrophone output was amplified additionally by $20 \mathrm{~dB}$ with a linear broadband amplifier (Ritec BR640, Warwick, RI, USA). The signals recorded by hydrophone were captured by 8 bit, $200 \mathrm{Ms}$ per s digital storage oscilloscope (HP54503A, Hewlett Packard, Colorado Springs, CO, USA), averaged over 16 consecutive waveforms, to maximize signal-to-noise-ratio, digitized, and then transferred to a personal computer (PC) for FFT (spectral) processing.

\section{Methodology of measurements in water}

The transmitting transducer was mounted in the $x y z$-axis positioning system driven by three stepper motors allowing its motion in the $x, y$ and $z$ directions with steps adjustable from 0.1 to $5 \mathrm{~mm}$ by PC. The transducer generated tone bursts with initial pressure amplitude of three different levels $P_{0}=37,103$ and $113 \mathrm{kPa}$ and corresponding to the weak and moderate nonlinear propagation condition. The measuring hydrophone was fixed.

The measurement results were compared with those simulated numerically for the source boundary conditions determined experimentally. As noted above, the radiating aperture apodization function was described by the analytical function $P_{0}(r)=P_{0} \mid 1-$ $\left(r / a_{\text {eff }}\right)^{10} \mid$, where the effective aperture radius $a_{\text {eff }}$ was found to be $14.5 \mathrm{~mm}$. When this function was assumed the radial pressure distribution calculated at the axial distance of $5 \mathrm{~mm}$ from the transducer face was best fitted to the measured one at the same distance. The initial pressure-time waveform of the generated tone bursts was described by the polynomial function

$$
P(t)=\left[1-\left|\left(t-t_{c}\right) /\left(t_{b}-t_{e}\right)\right|^{8} \cdot \sin \left[\omega\left(t-t_{c}\right)\right]\right.
$$

for $t_{b} \leq t \leq t_{e} ; P(t)=0$ for $t \notin\left(t_{b}, t_{e}\right)$. This function provided the best fitting between the simulated and measured waveform of the tone bursts generated by the transducer used.

\section{Measurement results in water}

The measurement results were compared with those simulated numerically using the model based on the TAWE approach. Figure 2 shows the axial peakcompression $\left(P^{+}\right)$, peak-rarefaction $\left(P^{-}\right)$and peakpeak $\left(P_{p p}\right)$ pressure variations in the pulsed focused nonlinear sound beams in water calculated for 8-cycle tone bursts with the initial pressure amplitude varied from $50 \mathrm{kPa}$ to $200 \mathrm{kPa}$ that corresponded to the weak or moderate source-pressure levels.
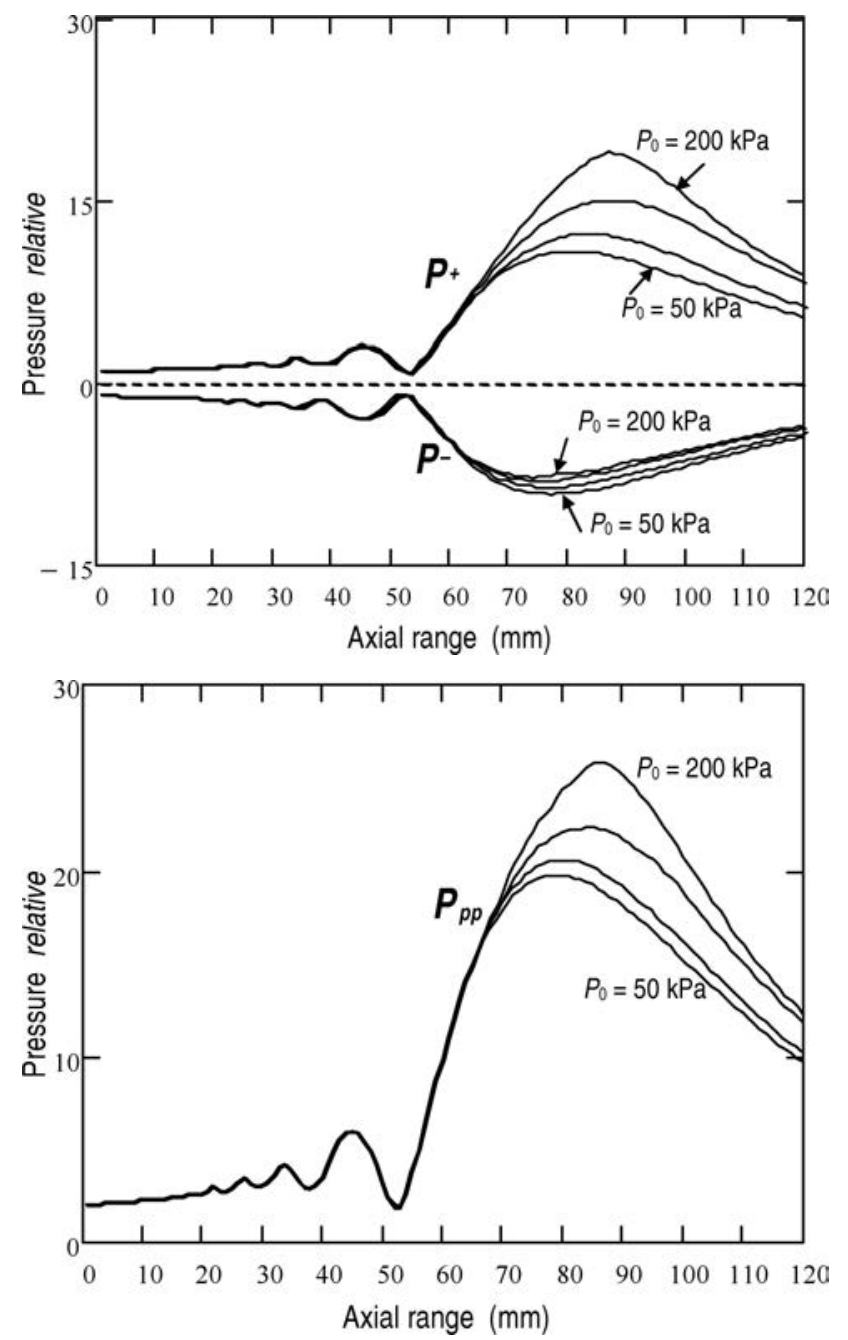

Fig. 2. Axial variations of the peak-compression $\left(P^{+}\right)$, peak-rarefaction $\left(P^{-}\right)$and peak-peak $\left(P_{p p}\right)$ pressure amplitude in pulsed focused nonlinear beams generated from the transducer used in water. Numerical simulations for 2.25 MHz 8-cycle tone bursts with the initial pressure amplitude varied from 50 to $200 \mathrm{kPa}$ with a $50 \mathrm{kPa}$ increment are shown.

These results indicate a correlation between the source-pressure amplitude and the focal plane position in the focused nonlinear beams. The higher is the source-pressure amplitude the closer to the geometric focus the true focal plane is positioned. The reason of this phenomena is the presence of a large amount of harmonics in the focal plane of the nonlinear beams.

The comparison of the measured and simulated axial peak-peak pressure variations in the pulsed focused nonlinear beams in water for three different levels of transducer excitation, providing the initial pressure amplitude of the generated tone bursts equal to 37, 103 and $113 \mathrm{kPa}$ is shown in Fig. 3. The obtained results have shown a very good agreement between the measurement data and the numerical predictions up to the axial distance of about $90 \mathrm{~mm}$ (focal distance for the nonlinear propagation mode) where the dis- 


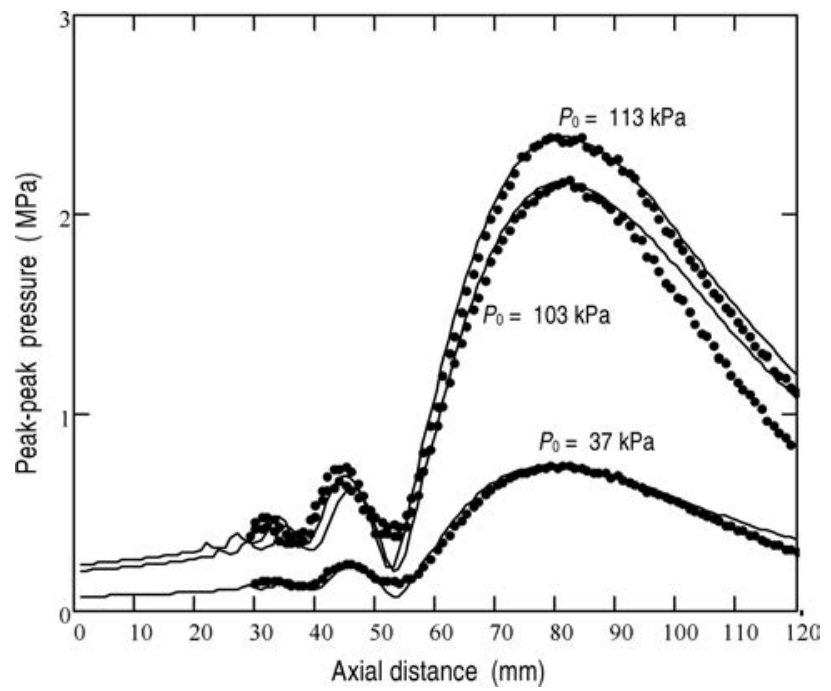

Fig. 3. Axial peak-peak pressure variations in the pulsed focused nonlinear acoustic beams produced by the transducer used generating in water 8-cycle tone bursts with the initial pressure amplitude varied between 0.037 and $0.113 \mathrm{MPa}$. Measurement data (points) and numerical simulations (solid lines) obtained for the source boundary conditions determined experimentally.

crepancy begins to be visible. Probable reason for this discrepancy is that the true plane wave conditions beyond the focal plane are not fulfilled.

Figure 4 illustrates the axial pressure variations of the fundamental (1st), 2nd and 3rd harmonics calculated for the tone bursts with the initial pressure amplitude varied from $50 \mathrm{kPa}$ to $200 \mathrm{kPa}$ generated from the transducer used here and propagating in water. It is evident from Fig. 4 that the axial distance from the transducer surface at which sudden growth of the second or higher harmonics amplitude appear is specific for this transducer regardless of the source-pressure.
The axial pressure variations of the fundamental and 2nd harmonics, measured for the tone bursts with various initial pressure amplitude $(37 \mathrm{kPa}$ and $113 \mathrm{kPa}$ ) generated from the transducer used here and propagating in water, are shown in Fig. 5 in comparison with those calculated under experimental boundary conditions. The distributions of predicted and measured pressure amplitudes of both the fundamental and 2nd harmonic components are virtually identical up to the axial distance of $90 \mathrm{~mm}$ (focal distance for the nonlinear propagation mode) where the discrepancy begins to be visible. Although the exact reason for this discrepancy is difficult to determine, it is conceivable that the true plane wave conditions are not fulfilled beyond the focal distance.

The quantitative analysis of the axial pressure variations of the fundamental and higher harmonics in weak to moderate nonlinear beams, produced in water by circular planar or focused transducers with $k a \gg 1$, has shown that the axial distance at which sudden growth of the 2nd or higher harmonics amplitude occurs is constant and specific for each transducer regardless of the source-pressure level. For the transducer considered here the axial distance at which sudden accretion of the 2nd harmonics amplitude occurs was found to be $60 \mathrm{~mm}$.

This characteristic feature of the weak and moderate pulsed nonlinear acoustic beams generated from axially-symmetric sources in water was used to develop an alternative semi-empirical method for determination of the nonlinearity parameter $B / A$ of clinically relevant media (KUJAWSKA et al., 2011a, 2011c).

Good agreement between measurement data and theoretical predictions in water confirmed applicability of the numerical model based on the TAWE method as an effective research tool for predicting of the $3 \mathrm{D}$ (2D space + time) pulsed nonlinear acoustic pressure fields produced by circular focused sources in water.
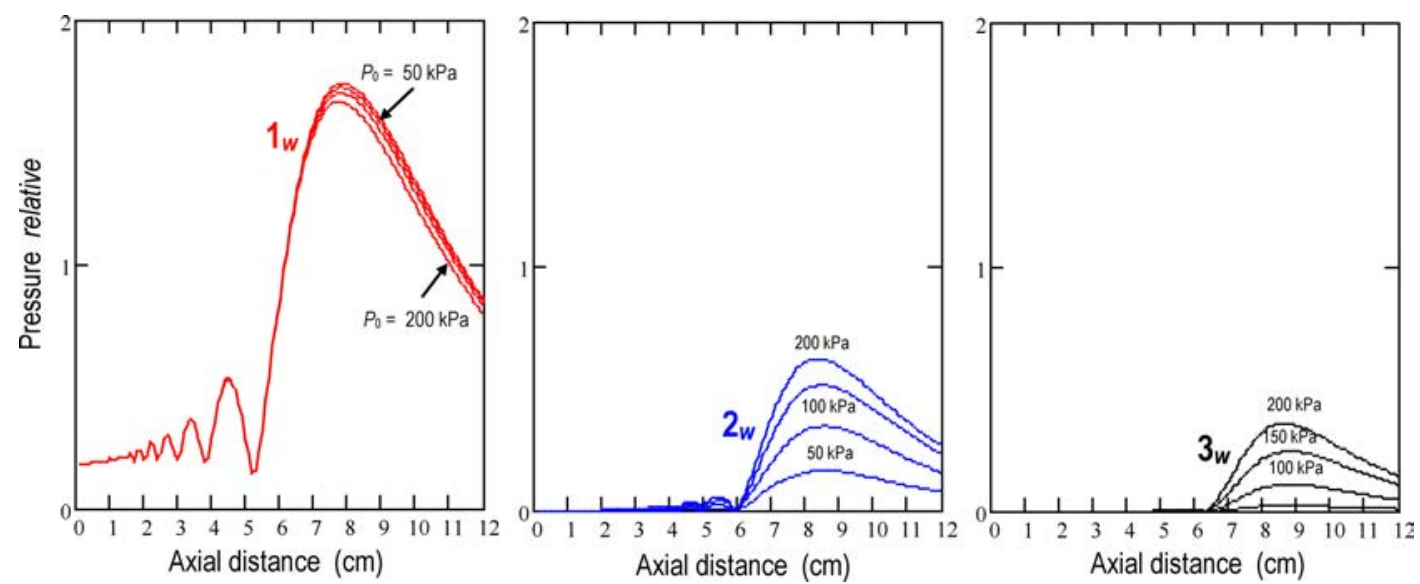

Fig. 4. Axial pressure variations of the fundamental $\left(1_{w}\right)$, 2nd $\left(2_{w}\right)$ and $3 r d(3 w)$ harmonics in the pulsed focused nonlinear acoustic beams produced by the transducer used generating in water $2.25 \mathrm{MHz} 8$-cycle tone bursts with the initial pressure amplitude varied between $50 \mathrm{kPa}$ and $200 \mathrm{kPa}$ with an increment of $50 \mathrm{kPa}$. 

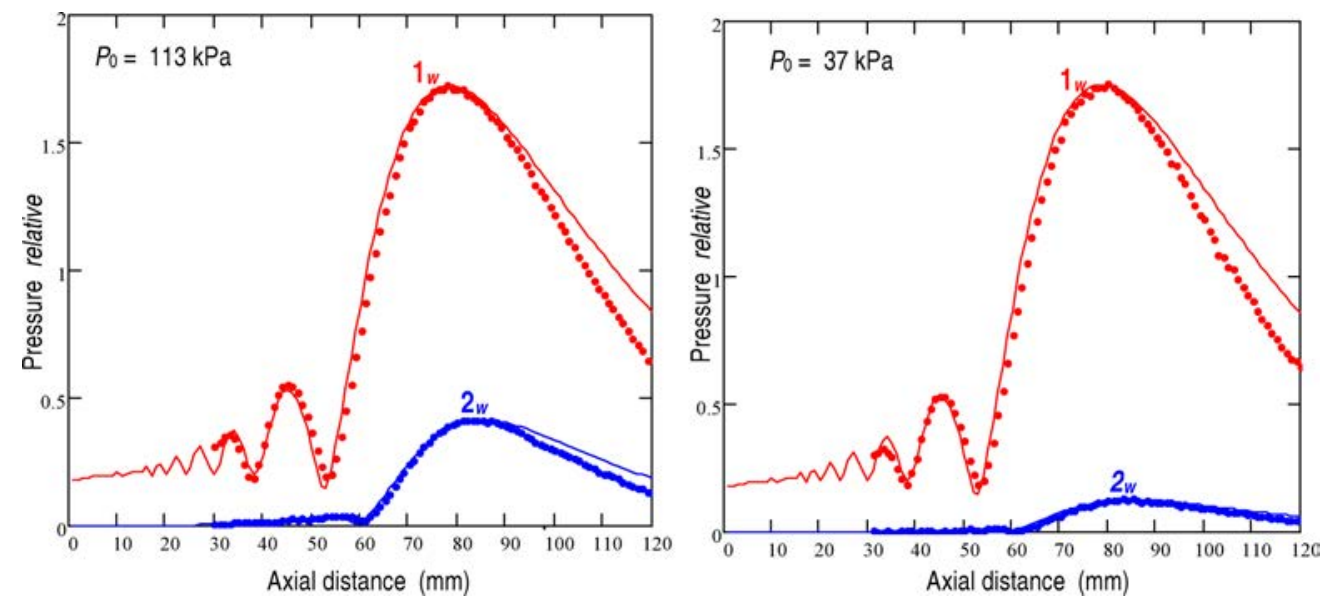

Fig. 5. Axial pressure variations of the fundamental $\left(1_{w}\right)$ and 2 nd $\left(2_{w}\right)$ harmonic amplitudes in the pulsed focused nonlinear acoustic beams produced by the transducer used generating in water 8-cycle tone bursts with the initial pressure amplitude of $113 \mathrm{kPa}$ (left) and $37 \mathrm{kPa}$ (right). Numerical simulation (solid lines) and measurement (points) results.

\section{Experimental setup for measurements in two-layer media}

Next, the model was verified experimentally for two-layer parallel media comprising of a $60-\mathrm{mm}$ water layer and a 60 -mm layer of 1.3-butanediol (99\%, Sigma-Aldrich Chemie GmbH, Steinheim, Germany) selected because of its tissue-mimicking acoustic properties and known nonlinearity parameter $B / A$. The thickness of the water layer was chosen to be equal to the axial distance at which sudden accretion of the 2nd harmonics amplitude occurs for the tone bursts propagating in water in order to maximize nonlinear effects in 1.3-butanediol. The path-length that tone bursts propagate through a 1.3-butanediol was chosen to be equal also to $60 \mathrm{~mm}$, however for strongly attenuating media in which harmonics are quickly suppressed this path-length may be chosen shorter.

All measurements in the two-layer media: water/1.3-butanediol were carried out at a $25{ }^{\circ} \mathrm{C}$ temperature using the experimental setup shown in Fig. 1. In order to determine experimentally the harmonic pressure variations in the pulsed nonlinear acoustic beam produced in the two-layer media the two-chamber coaxial design was immersed in the larger, temperature controlled water tank. The fixed length $(L=60 \mathrm{~mm})$ chamber was filled with distilled water. Its proximal end was sealed by the $2.25 \mathrm{MHz}$ transducer used. The distal end boundary was made from acoustically transparent 20- $\mu \mathrm{m}$ thick Mylar film. The diameter of the fixed-length chamber was slightly smaller than that of the variable-length one, so the fixed-length chamber could be moved coaxially in such a way that it varied the length of the second chamber. The second chamber had independent inlets, so some volume of the 1.3-butanediol could be removed when the first chamber was moved towards the hydrophone. The distal end of the variable-length chamber was also sealed with 20- $\mu \mathrm{m}$ thick Mylar film. The $x y z$ micromanipulator was PC controlled and allowed recording of the pressure-time waveforms versus axial distance. The sensitive element of the stationary broadband bilaminar membrane hydrophone (Sonora, SN S5-259) used to record the $P(t)$ waveforms was positioned at the axial distance of $1.7 \mathrm{~mm}$ behind the 2 nd chamber Mylar window. The maximal path-length of the two chambers was $120 \mathrm{~mm}$ (fixed-length chamber $60 \mathrm{~mm}$ and variable-length chamber $60 \mathrm{~mm}$ ). So, the data presented in the next section were recorded starting at the axial distance of $120 \mathrm{~mm}$ from the transducer face and ending at the axial distance of $80 \mathrm{~mm}$, which was in the vicinity of the focal plane of the source for the linear propagation mode.

\section{Measurement results in two-layer media}

The measurement results in the two-layer media comprising of a $60 \mathrm{~mm}$ water layer and a $60 \mathrm{~mm}$ layer of 1.3-butanediol were compared with the results predicted numerically for the assumed source boundary conditions determined experimentally. Figure 6 shows the comparison between the measured axial pressure distributions of the fundamental (1st), 2nd and 3rd harmonics in the pulsed nonlinear acoustic beam produced in the two-layer configuration of media and their numerical predictions obtained using the TAWE approach for the transducer used. In Fig. 6 the fundamental, 2nd and 3rd harmonic components measured along the beam axis are plotted for the axial distance varying from $80 \mathrm{~mm}$ to $120 \mathrm{~mm}$. The agreement between the simulation and measurement results at the axial distances up to $90 \mathrm{~mm}$ (focal distance for the nonlinear propagation mode) is very good, however at the distances larger than $90 \mathrm{~mm}$ the discrepancy begins to be visible. The exact reason for this discrepancy is difficult to determine. It is likely that the discrepancy 

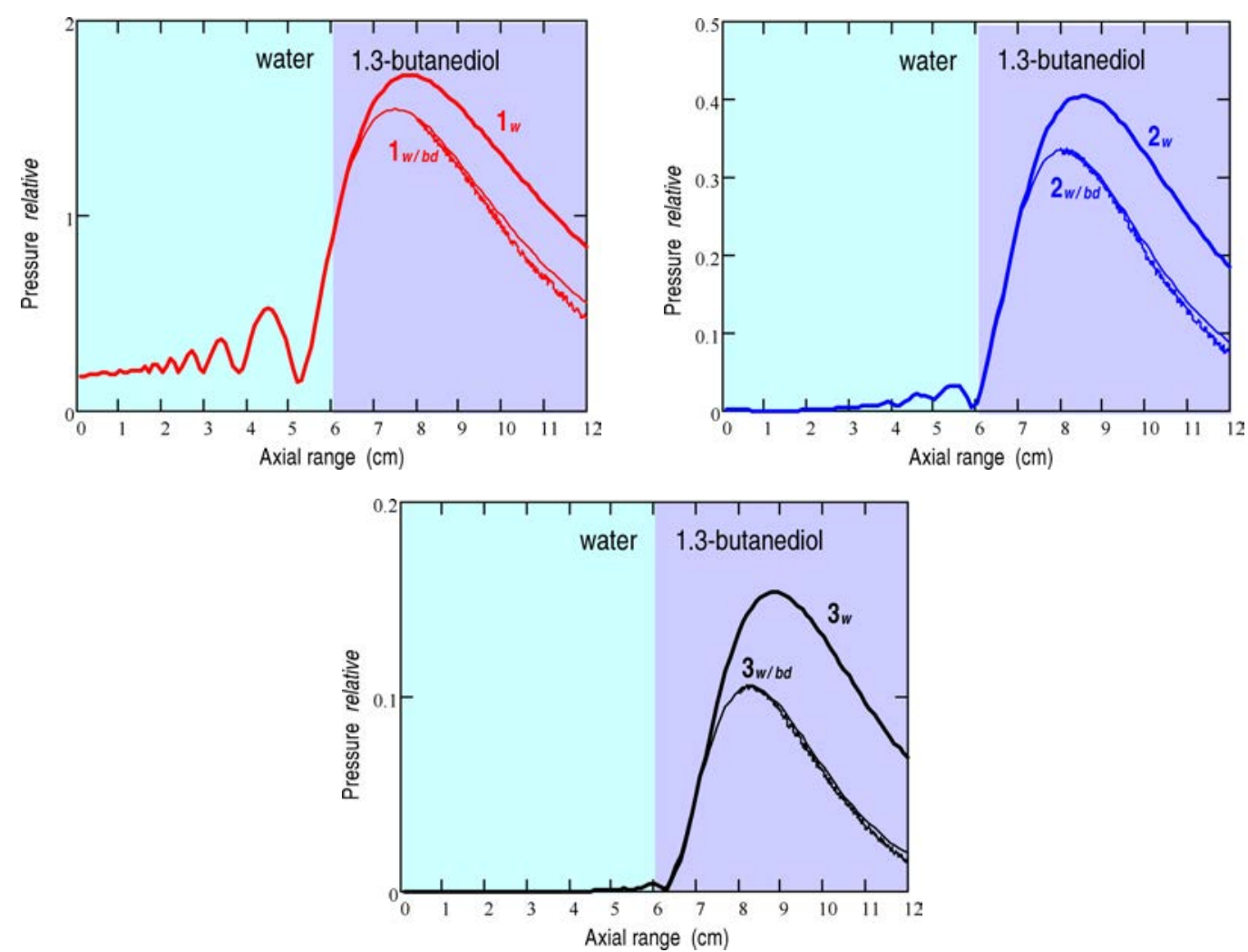

Fig. 6. Axial pressure variations of the fundamental (1), 2nd (2) and 3rd (3) harmonics in the pulsed focused nonlinear beam produced by the transducer used in water (thick lines, subscripts $w$ ) and in two-layer media comprising of a $60-\mathrm{cm}$ water layer and a 60-cm layer of 1.3-butanediol (thin lines, subscripts $w / b d$ ). Calculation (solid lines) and measurement

(points) results for $2.25 \mathrm{MHz}$ 8-cycle tone bursts with the initial pressure amplitude of $117 \mathrm{kPa}$ are shown.

is due to the fact that the plane wave conditions beyond the focal plane are not met. The results presented in Fig. 6 demonstrate that the nonlinear propagation model based on the TAWE approach is capable of predicting the pulsed nonlinear acoustic pressure fields from axially-symmetric sources in layered media with attenuation arbitrarily dependent on frequency. So, the model can be used as an effective research tool for prediction of both the 3D acoustic pressure variations and heat sources distributions (induced due to these fields) in pulsed focused nonlinear beams produced by clinically relevant therapeutic sources in layered media comprising of physiological fluids and tissues.

Figures 7, 8, 9 and 10 show the axial pressure variations of the fundamental (1st), 2nd and 3rd harmonic components in the pulsed nonlinear beams generated
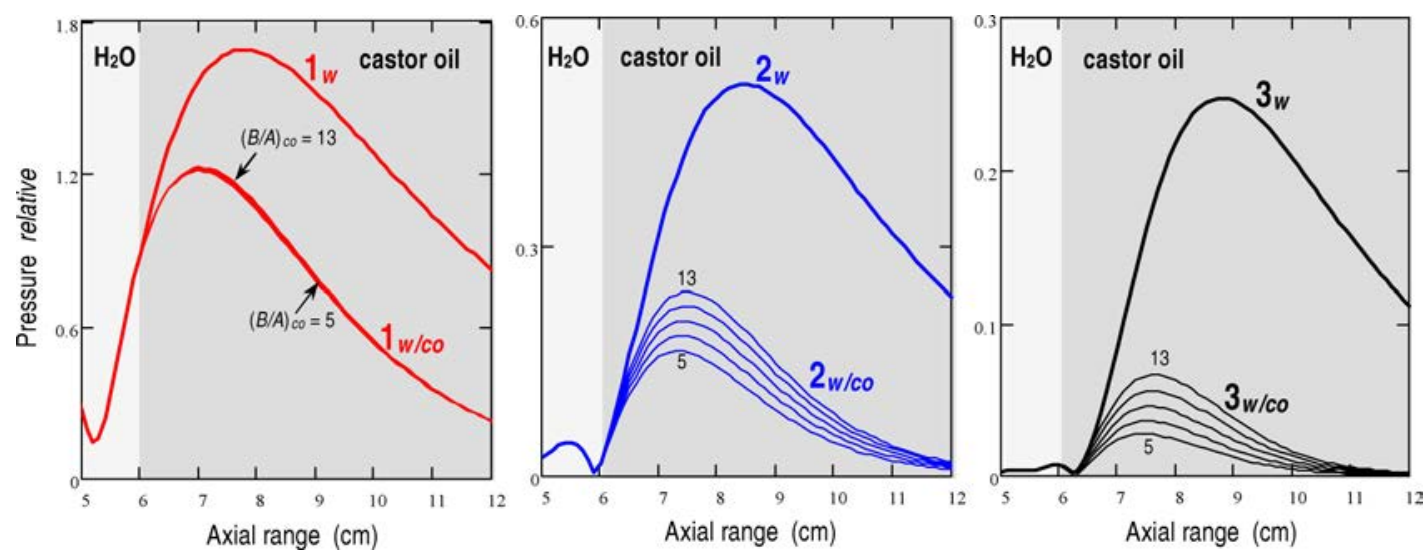

Fig. 7. Axial pressure variations of the fundamental (1st), 2nd (2) and 3rd (3) harmonics in the pulsed focused nonlinear beam produced by the transducer used generating 8-cycle tone bursts with the initial pressure amplitude of $0.15 \mathrm{MPa}$ in water (subscripts $w$ ) and in two-layer media (subscripts $w / c o$ ) comprising of a 6-cm water layer and a 6 -cm layer of castor oil whose value of the $(B / A)_{c o}$ assumed in calculations was varied between 5 and 13 with an increment of 2 . Pressure variations are shown at the axial range between $5 \mathrm{~cm}$ and $12 \mathrm{~cm}$. 
from the transducer used and calculated for 8-cycle tone bursts with the initial pressure of $150 \mathrm{kPa}$ propagating in water or in two-layer media: 6 -cm water $/ 6-\mathrm{cm}$ tested medium (castor oil, silicone oil, pig liver and human brain, respectively) whose value of the $B / A$ pa- rameter assumed in calculations was varied between 5 and 13 with various increments.

The linear and nonlinear acoustic parameters assumed in calculations for media considered are quoted in Table 1.
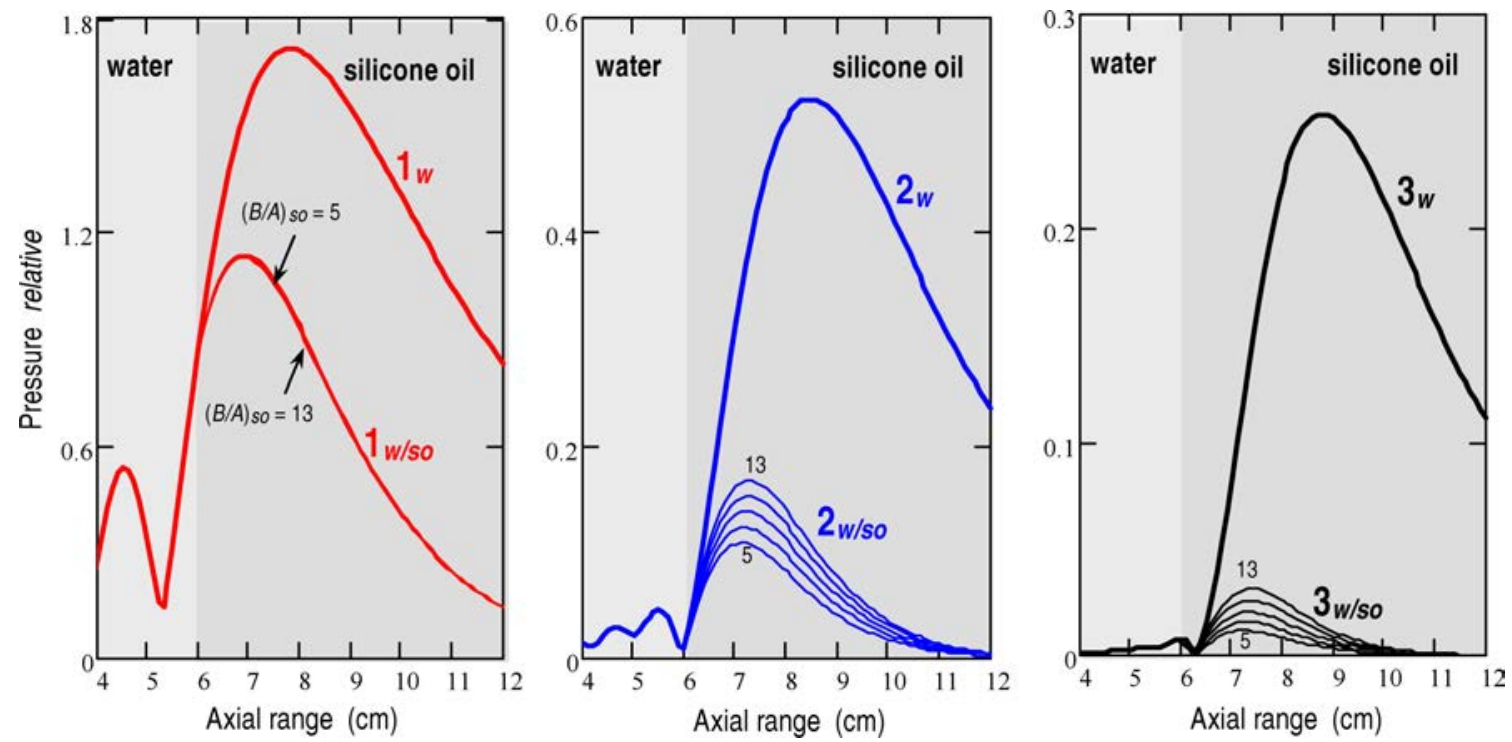

Fig. 8. Axial pressure variations of the fundamental (1), 2nd (2) and 3rd (3) harmonics in the pulsed focused nonlinear beam produced by the transducer used generating 8-cycle tone bursts with the initial pressure amplitude of $0.15 \mathrm{MPa}$ in water (subscripts $w$ ) and in two-layer media (subscripts $w / s o$ ) comprising of a 6 -cm water layer and a 6 -cm layer of silicone oil. The nonlinearity parameter $(B / A)_{\text {so }}$ assumed in calculations for silicone oil was varied between 5 and 13 with an increment 2. Pressure variations of harmonics are shown at the axial range between $4 \mathrm{~cm}$ and $12 \mathrm{~cm}$.
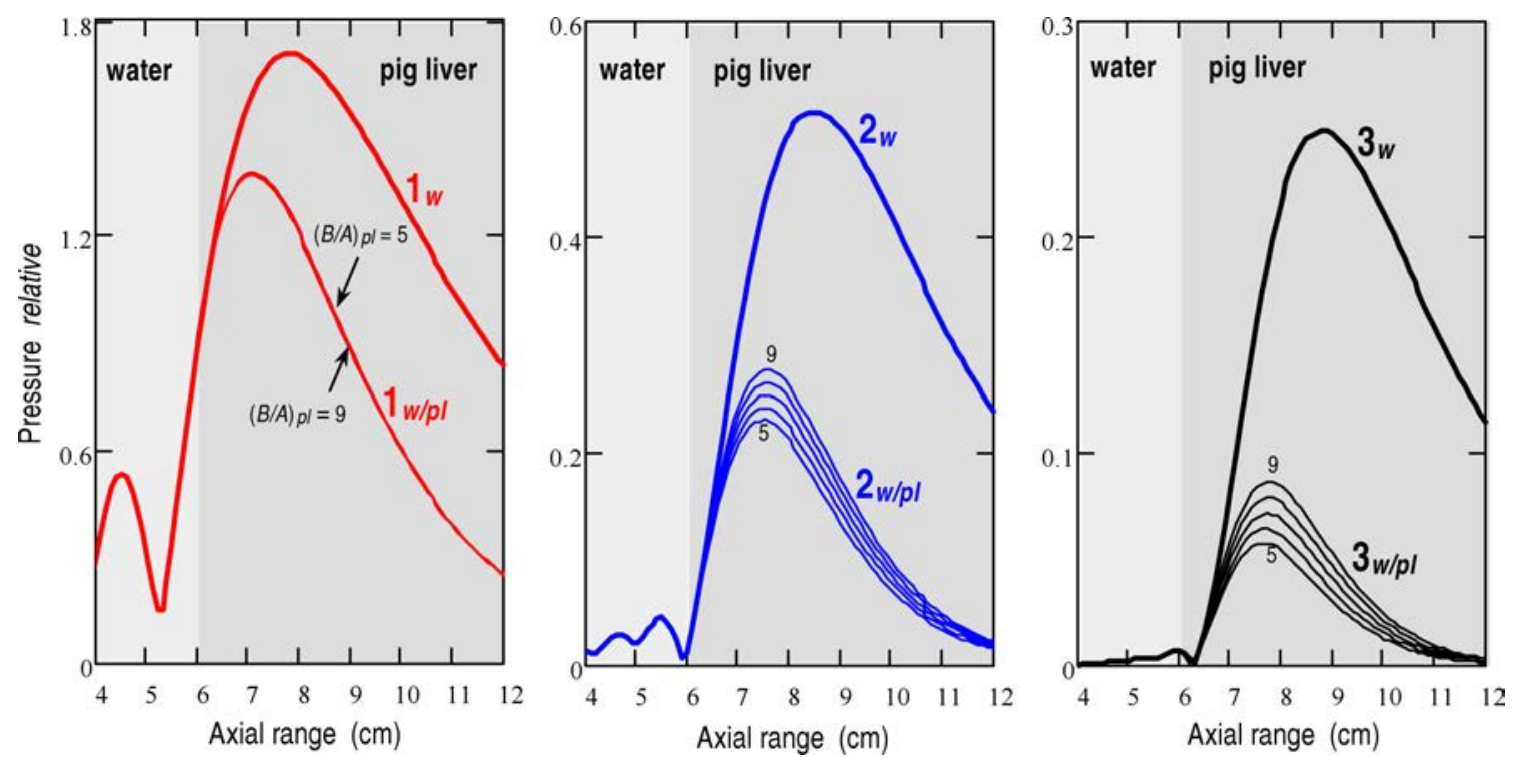

Fig. 9. Axial pressure variations of the fundamental (1), 2nd (2) and 3rd (3) harmonics in the pulsed focused nonlinear beam produced by the transducer used generating 8-cycle tone bursts with the initial pressure amplitude of $0.15 \mathrm{MPa}$ in water (subscripts $w$ ) and in two-layer media (subscripts $w / p l$ ) comprising of a 6 -cm water layer and a 6 -cm layer of pig liver whose value of the $(B / A)_{p l}$ assumed in calculations was varied between 5 and 9 with an increment 1. 

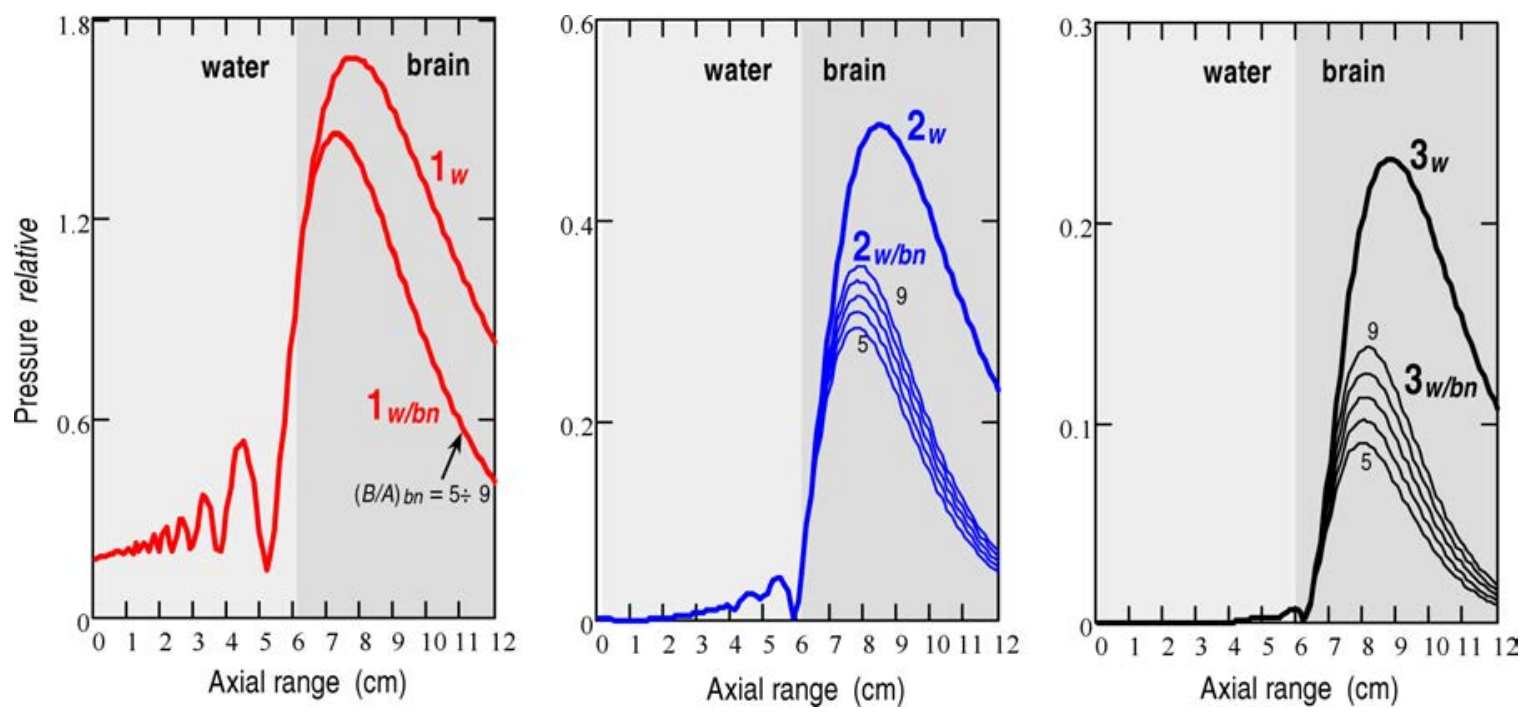

Fig. 10. Axial pressure variations of the fundamental (1), 2nd (2) and 3rd (3) harmonics in the pulsed focused nonlinear beam from the transducer used generating 8-cycle tone bursts with the initial pressure amplitude of $0.15 \mathrm{MPa}$ in water (subscripts $w$ ) and in two-layer media (subscripts $w / b n$ ) comprising of a 6 -cm water layer and a 6 -cm layer of human brain whose value of the $(B / A)_{b n}$ assumed in calculations was varied between 5 and 9 with an increment 1 .

\section{Conclusions}

The results of the measurements of pulsed focused nonlinear acoustic fields in water and in two-layer media: water/1.3-butanediol produced by the $2.25 \mathrm{MHz}$ circular focused (f-number 3 ) transducer with diameter of $29 \mathrm{~mm}$ were compared with the numerical predictions obtained using the Time-Averaged Wave Envelope (TAWE) approach to the numerical solution of the second order nonlinear differential wave equation for axially-symmetric sources (WóJCIK et al., 2006). The comparison of the experimental results and numerical predictions has shown that the TAWE approach is capable of predicting fairly well both the spatial-peak and spatial-spectral pressure distributions in pulsed focused nonlinear beams produced in layered biological media by clinically relevant therapeutic sources.

Discrepancies between the theoretically predicted axial pressure distributions and those determined experimentally are visible beyond the focal plane. It is conceivable that the true plane wave conditions are not fulfilled in this region. The data presented verified the applicability of the TAWE method as an effective research tool for prediction both the pulsed focused nonlinear acoustic pressure fields and heat sources distributions from clinically relevant therapeutic probes in layered media comprising of physiological fluids and tissues. Such effective research tool is indispensable for planning noninvasive ultrasonic therapies in layered soft tissues and especially inside a brain when the nonlinear pulsed waves have to pass through a skull bone strongly attenuating ultrasonic waves.

\section{Acknowledgment}

This work was supported by the Ministry of Science and Higher Education from means on the statutory activity and by the National Science Centre from the Grant 2011/01/B/ST7/06735.

\section{References}

1. Baker A.C., Anastasiadis K., Humphrey V.F. (1988), The nonlinear pressure field of a plane circular piston: theory and experiment, J. Acoust. Soc. Am., 84, 1483-1487.

2. Beyer R.T. (1997), The B/A parameter, [in:] Nonlinear acoustics, Hamilton M.F., Blackstock D.T. [Eds.], Academic Press, NY, pp. 25-37.

3. Chavrier F., Lafon C., Birer A., Barriere C., Jacob X., Cathignol D. (2006), Determination of the nonlinear parameter by propagating and modelling finite amplitude plane waves, J. Acoust. Soc. Am., 119, 5, 2639-2644.

4. Duck F.A. (1990), Physical Properties of Tissue, Academic Press, London.

5. Kujawska T., Nowicki A., Lewin P.A. (2011a), Determination of nonlinear medium parameter $B / A$ using model assisted variable-length measurement approach, Ultrasonics, 51, 997-1005.

6. Kujawska T., Secomski W., Krawczyk K., NoWICKI A. (2011b), Thermal effects induced in liver tissues by pulsed focused ultrasonic beams from annular array transducer, Archives of Acoustics, 36, 937-944.

7. Kujawska T., Wójcik J., Nowicki A. (2011c), Determination of the $B / A$ of biological media by measuring and modeling nonlinear distortion of pulsed acoustic wave in two-layer system of media, [in:] Acousti- 
cal Imaging, vol. 30, 295-303, M. Andre et al. [Eds.], Springer Science \& Business Media B.V.

8. Nachef S., Cathignol D., Tuøtta J.N., Berg A.M., ТЈøтта S. (1995), Investigation of a high intensity sound beam from a plane transducer. Experimental and theoretical results, J. Acoust. Soc. Am., 98, 4, 23032323.

9. National Physical Laboratory, Kaye\&Laby, Tables of Physical \& Chemical Constants, Medical Ultrasonics, http://www.kayelaby.npl.co.uk.
10. Wójcik J., Nowicki A., Lewin P.A., Bloomfield P.E., Kujawska T., Filipczyński L. (2006), Wave envelopes method for description of nonlinear acoustic wave propagation, Ultrasonics, 44, 310-329.

11. Wójcik J., Kujawska T., Nowicki A., Lewin P.A. (2008), Fast prediction of pulsed nonlinear acoustic fields from clinically relevant sources using TimeAveraged Wave Envelope approach: comparison of numerical simulations and experimental results, Ultrasonics, 48, 707-715. 